\title{
HST-STIS abundances in the uranium-rich very metal-poor star CS 31082-001
}

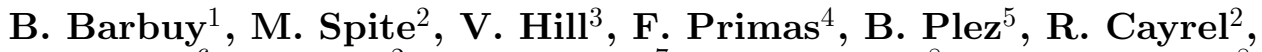 \\ C. Sneden ${ }^{6}$, F. Spite ${ }^{2}$, T. C. Beers ${ }^{7}$, J. Andersen ${ }^{8}$, B. Nordström ${ }^{8}$, \\ P. Bonifacio ${ }^{2}$, P. François ${ }^{2}$, P. Molaro ${ }^{9}$, and C. Siqueira-Mello ${ }^{1}$ \\ ${ }^{1}$ Universidade de São Paulo, Brazil \\ ${ }^{2}$ Observatoire de Paris, France \\ ${ }^{3}$ Observatoire de la Côte d'Azur, France \\ ${ }^{4}$ European Southern Observatory, Germany \\ ${ }^{5}$ Université de Montpellier, France \\ ${ }^{6}$ University of Texas, USA \\ ${ }^{7}$ Michigan State University, USA \\ ${ }^{8}$ Niels Bohr Institute, Denmark \\ ${ }^{9}$ Osservatorio Astronomico di Trieste, Italy
}

\begin{abstract}
The abundance derivation of heavy r-elements may provide a better understanding of the r-process, and the determination of several reference r-elements should allow a better determination of the star's age. The spatial ultraviolet (UV) region presents a large number of lines of heavy elements, and in some cases such as $\mathrm{Bi}, \mathrm{Pt}, \mathrm{Au}$, detectable lines are only available in the UV. The extreme "r-process star" CS 31082-001 $([\mathrm{Fe} / \mathrm{H}]=-2.9)$ was observed in the spatial UV in order to determine abundances of the heavy elements, using STIS on board HST.
\end{abstract}

Keywords. metal-poor stars, r-elements, ultraviolet

\section{Introduction}

The ESO large programme FIRST STARS was devoted to the accurate determination of abundances in very metal-poor stars. A very interesting star was revealed (Hill et al. 2002; Cayrel et al. 2001), to be extremely enhanced in r-process elements, in particular two radioactive elements of the third peak of the r-process: thorium and uranium. The uranium line at $3859.6 \AA$ was measurable, so that the ratio of the abundances of $\mathrm{Th}$ and $\mathrm{U}$ was well determined, and could thus provide a particularly reliable determination of the age of this star. Indeed, possible variations of the r-process will affect much less the ratio of these two elements, which have very similar atomic and nuclear structures. It is however desirable to check the decay of the radioactive elements by comparison with other stable elements of the third r-process peak, together with $\mathrm{Pb}$, a key element (Plez et al. 2004), and elements of the second peak. Besides CS 31082-001 and CS 22892-052 (Sneden et al. 1994), Frebel et al. (2007) reported the identification of another such star, $\mathrm{HE}$ 1523-0901. This latter object has $\mathrm{V}=11.1,[\mathrm{Fe} / \mathrm{H}]=-2.95,[\mathrm{r} / \mathrm{Fe}] \sim 1.8$, and an age of 13.2 Gyr was deduced from the U/Th, U/Ir, Th/Eu, and Th/Os ratios.

\section{Avenue for progress}

The main aim now is to reduce the errors in the age derivation terms of $\Delta \mathrm{t}(\mathrm{Gyr})=21.8$ $\left[\log (\mathrm{U} / \mathrm{Th})_{\text {init }}-\log (\mathrm{U} / \mathrm{Th})_{\text {now }}\right]$. New calculations by Toenjes et al. (2001) predict log 
Table 1. Derived abundances.

\begin{tabular}{lcccc}
\hline Element & Line $[\AA]$ & $\mathbf{Z}$ & $\mathbf{A}($ Sun $)$ & $\mathbf{A}($ Star) \\
\hline Os I & 2838.6 & 76 & 1.45 & 0.10 \\
Os I & 3058.6 & 76 & 1.45 & 0.30 \\
Pt I & 2929.8 & 78 & 1.80 & 0.65 \\
Pt I & 3064.7 & 78 & 1.80 & 0.40 \\
Au I & 2675.9 & 79 & 1.01 & -0.90 \\
Pb I & 2833.0 & 82 & 1.95 & -0.65 \\
Bi I & 3067.7 & 83 & 0.68 & -0.50 \\
\hline
\end{tabular}

$(\mathrm{U} / \mathrm{Th})_{\text {init }}=-0.16$, but should improve with the knowledge of other r-process elements involved in the third peak, where Lead and Bismuth are the obvious targets. Plez et al. (2004) were able to measure the $\mathrm{Pb}$ abundance in CS 31082-001 from near-UV spectra obtained with the VLT-UVES at ESO. An abundance of $\log (\mathrm{Pb} / \mathrm{H})+12=-0.55$ was obtained, very close to the value of -0.73 , expected from the decay of ${ }^{238} \mathrm{U}$ and ${ }^{232} \mathrm{Th}$ alone. This low value of $\mathrm{Pb}$ leaves little room for an s-process contribution in the solar system.

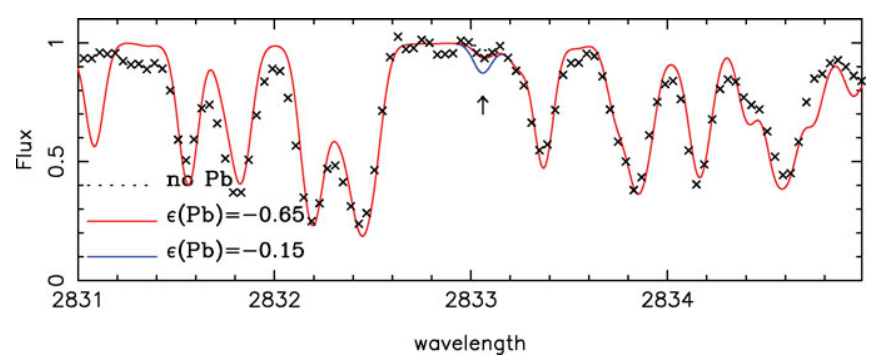

Figure 1. Pb 2833.053 Å line: crosses: observed spectrum, solid red and blue lines: synthetic spectra computed with $[\mathrm{Pb} / \mathrm{Fe}]=$ none, $-0.65,-0.15$.

The code turbospectrum (Alvarez \& Plez 1998) was employed to compute synthetic spectra in the UV region. In this spectral region it is crucial to properly account for scattering in the continuum. So far, we have derived abundances for the elements shown in Table 1. We confirm that the Pb abundance in CS 31082-001 is low, whereas only upper limits are available for CS 22892-052 and HE 1523-0901. Os and Pt are enhanced, as $\mathrm{U}$ and $\mathrm{Th}$, whereas $\mathrm{Bi}$ and $\mathrm{Au}$ are less enhanced. Further abundances are being derived for other elements (GdII, DyII, ErII, LuII among others).

Fig. 1 shows the fit of synthetic spectra to the observed $\mathrm{Pb} 2833.052 \AA$ line.

\section{References}

Alvarez, R. \& Plez, B. 1998, A\&A, 330, 1109

Cayrel, R., Hill, V., Beers, T. C., Barbuy, B, Spite, M. et al. 2001, Nature, 409, 691

Hill, V., Plez, B., Cayrel, R., Beers, T. C., Nordström, B. et al. 2002, A\& A, 387, 560

Plez, B., Hill, V., Cayrel, R., Spite, M., Barbuy, B. et al. 2004, A\&A A, 428, L9

Sneden, C., Preston, G. W., McWilliam, A., \& Searle, L. 1994, ApJ, 431, L27

Toenjes, R., Schatz, H., Kratz, K.-L., Pfeiffer, B., Beers, T. C. et al. 2002, ASPC, 245, 376 